\title{
Structure-function analysis of yeast tRNA ligase
}

\author{
LI KAI WANG and STEWART SHUMAN
}

Molecular Biology Program, Sloan-Kettering Institute, New York, New York 10021, USA

\begin{abstract}
Trl1 is an essential 827-amino-acid enzyme that executes the end-healing and end-sealing steps of tRNA splicing in Saccharomyces cerevisiae. Trl1 consists of two catalytic domains-an N-terminal adenylyltransferase/ligase component (amino acids 1-388) and a C-terminal 5'-kinase/cyclic phosphodiesterase component (amino acids 389-827) — that can function in tRNA splicing in vivo when expressed as separate polypeptides. Sedimentation analysis indicates that the ligase and kinase/CPD domains are monomeric proteins that do not form a stable complex in trans. To understand the structural requirements for the RNA ligase component, we performed a mutational analysis of amino acids that are conserved in Trl1 homologs from other fungi. Alanine scanning identified 23 new residues as essential for Trl1-(1-388) activity in vivo. Structure-activity relationships at these positions, and four essential residues defined previously, were clarified by introducing 50 different conservative substitutions. Lethal mutations of Lys114, Glu184, Glu266, and Lys284 abolished Trl1 adenylyltransferase activity in vitro. The essential elements embrace (1) putative equivalents of nucleotidyltransferase motifs I, Ia, III, IV, and V found in DNA ligases, T4 RNA ligase 2, and mRNA capping enzymes; (2) an N-terminal segment shared with the T4 RNA ligase 1 subfamily only; and (3) a constellation of conserved residues specific to fungal tRNA splicing enzymes. We identify yeastlike tRNA ligases in the proteomes of Leishmania and Trypanosoma. These findings recommend tRNA ligase as a target for antifungal and antiprotozoal drug discovery.
\end{abstract}

Keywords: tRNA splicing; polynucleotide ligase; adenylyltransferase; antifungal target

\section{INTRODUCTION}

Yeast tRNA splicing occurs in three genetically and biochemically distinct stages (Abelson et al. 1998). First, a splicing endonuclease breaks the phosphodiester backbone of pretRNA at the exon-intron boundaries to yield $2^{\prime}, 3^{\prime}$ cyclic phosphate and $5^{\prime}-\mathrm{OH}$ termini at both incision sites. Second, the ends of the broken tRNA halves are healed and then sealed by yeast tRNA ligase (Trl1) to form a spliced tRNA containing a $2^{\prime}-\mathrm{PO}_{4}, 3^{\prime}-5^{\prime}$ phosphodiester at the splice junction. Third, the $2^{\prime}-\mathrm{PO}_{4}$ at the splice junction is removed by the $2^{\prime}$-phosphotransferase Tpt1.

The healing and sealing phases of the yeast tRNA splicing pathway consists of three enzymatic reactions catalyzed by Trl1: (1) the $2^{\prime}, 3^{\prime}$ cyclic phosphate terminus is hydrolyzed to a $3^{\prime}-\mathrm{OH}, 2^{\prime}-\mathrm{PO}_{4}$ terminus by a $2^{\prime}, 3^{\prime}$ cyclic phosphodiesterase (CPD) activity; (2) the $5^{\prime}-\mathrm{OH}$ terminus is phosphorylated by a GTP-dependent polynucleotide kinase activity; and (3) the resulting $3^{\prime}-\mathrm{OH}, 2^{\prime}-\mathrm{PO}_{4}$ and $5^{\prime}-\mathrm{PO}_{4}$ ends are sealed by an ATP-dependent RNA ligase activity (Greer et al. 1983; Apostol

Reprint requests to: Stewart Shuman, Molecular Biology Program, Sloan-Kettering Institute, New York, NY 10021, USA; e-mail: s-shuman@ ski.mskcc.org; fax (212) 717-3623.

Article and publication are at http://www.rnajournal.org/cgi/doi/ 10.1261/rna.2170305. et al. 1991; Westaway et al. 1993; Sawaya et al. 2003). Trl1 consists of an N-terminal ligase module, a central polynucleotide kinase module, and a C-terminal CPD module (Fig. 1). The mechanism of the ligase component of yeast tRNA ligase generally resembles that of bacteriophage T4 RNA ligase 1 (Rnl1), whereby RNA joining entails three nucleotidyl transfer steps: (1) ligase reacts with ATP to form a covalent ligase(lysyl-N)-AMP intermediate plus pyrophosphate; (2) AMP is transferred from ligase-adenylate to the $5^{\prime}-\mathrm{PO}_{4} \mathrm{RNA}$ end to form an RNA-adenylate intermediate (AppRNA); and (3) ligase catalyzes attack by an RNA $3^{\prime}-\mathrm{OH}$ on the RNA-adenylate to seal the two ends via a phosphodiester bond and release AMP (Cranston et al. 1974; Sugino et al. 1977). tRNA ligase and T4 Rnll are functionally and structurally homologous proteins (Sawaya et al. 2003; Wang et al. 2003) dedicated to the repair of programmed tRNA breaks in vivo (Amitsur et al. 1987; Phizicky et al. 1992). A key distinction between the two enzymes is that tRNA ligase apparently requires the terminal $2^{\prime}-\mathrm{PO}_{4}$ on the proximal tRNA halfmolecule, whereas T4 Rnll does not (Schwer et al. 2004).

tRNA ligase and Rnl1 exemplify a distinct branch of the covalent nucleotidyltransferase enzyme superfamily. The superfamily is composed of ATP-dependent and $\mathrm{NAD}^{+}$-dependent DNA ligases, ATP-dependent RNA ligases, and GTP-dependent mRNA capping enzymes, all of which catalyze the nucleotidylation of polynucleotide $5^{\prime}$ ends via a 


Ligase Kinase CPD
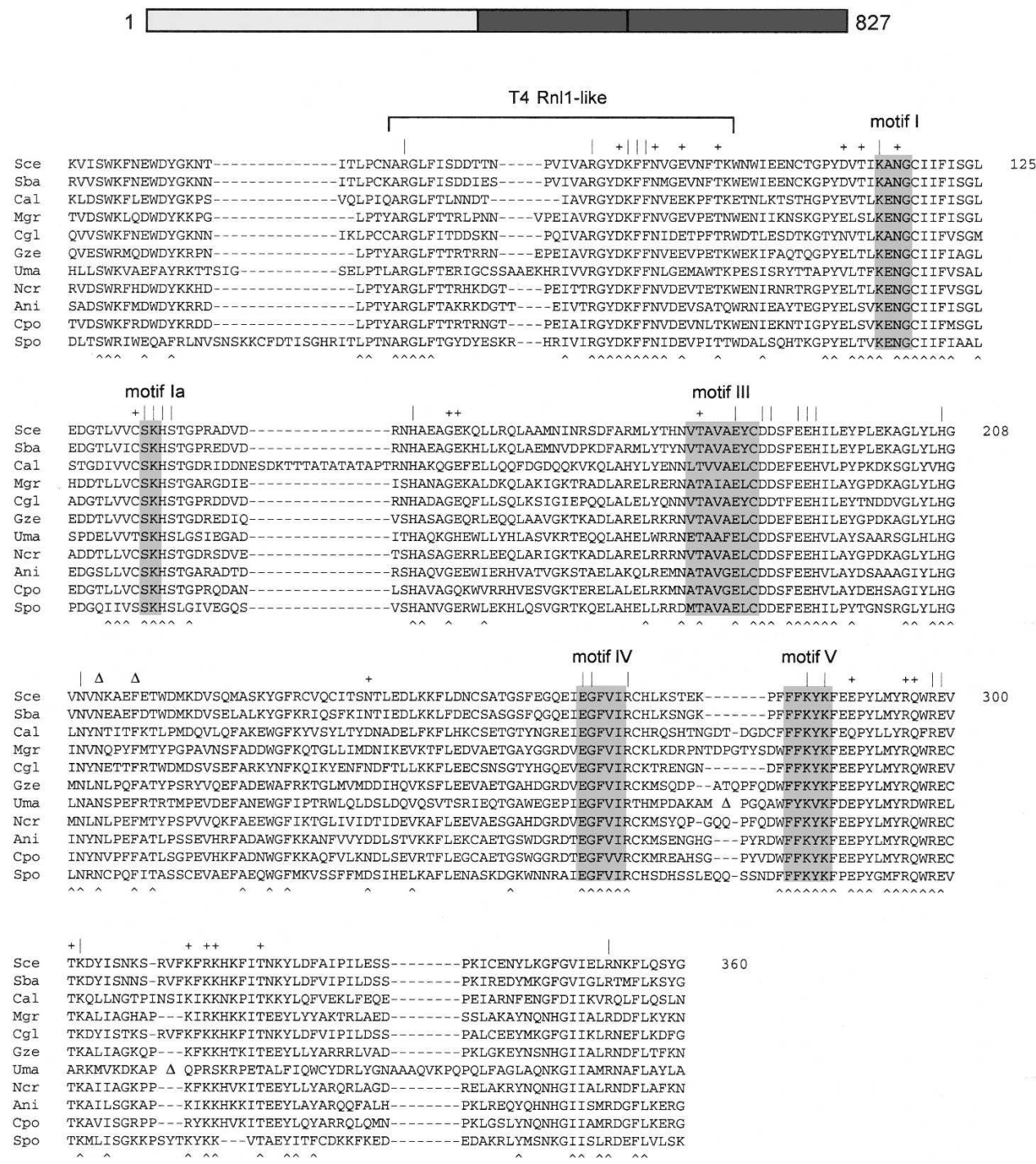

FIGURE 1. Fungal tRNA ligases. The N-terminal ligase domain and C-terminal kinase-CPD domain of Trll are depicted as light and dark horizontal bars. The amino acid sequence of the ligase domain of S. cerevisiae (Sce) Trl1 from residues 51-360 is aligned to the sequences of the homologous proteins of Saccharomyces bayanus (Sba), Candida albicans (Cal), Magnaporthe grisea (Mgr), Candida glabrata (Cgl), Gibberella zeae (Gze), Ustilago maydis (Uma), Neurospora crassa (Ncr), Aspergillus nidulans (Ani), Coccidioides posadasii (Cpo), and Schizosaccharomyces pombe (Spo). Positions of side chain identity or similarity in all 11 proteins are denoted by ' below the alignment. Nucleotidyltransferase motifs I, Ia, IV, and V and the newly identified motif III are highlighted in shaded boxes. Residues identified as essential by alanine scanning are indicated by $\mid$. Nonessential residues are indicated by + . Positions Asn 212 and Phe216 at which alanine mutations conferred a ts phenotype are indicated by $\Delta$ above the alignment.

covalent enzyme-(lysyl-Nל)-NMP intermediate (Shuman and Lima 2004). The typical nucleotide-binding pocket of covalent nucleotidyltransferases is composed of six peptide motifs (I, Ia, III, IIIa, IV, and V) that contribute essential constituents of the active site. Structure-function studies of T4 Rnl1 and yeast tRNA ligase identified the putative equivalents of motifs I, Ia, IV, and V (Fig. 1), but revealed no obvious counterparts of motifs III or IIIa, or of the OB-fold domain located immediately downstream of motif $\mathrm{V}$ in DNA ligases and mRNA capping enzymes (Sawaya et al. 2003; Wang et al. 2003). Thus, the evolutionary relationship between tRNA ligase and other covalent nucleotidyltransferases remains unclear.
The ligase/adenylyltransferase functions of Trl1 reside within the $\mathrm{N}$-terminal segment. Alignment of the $\mathrm{N}$-terminal domains of Trl1 orthologs from 11 species of fungi reveals 120 positions of identity/similarity in all 11 polypeptides (indicated by ${ }^{\wedge}$ in Fig. 1). It is remarkable that no homolog of the Trl1 ligase domain can be detected in the available proteomes of any metazoan species, which makes tRNA ligase a plausible target for antifungal drug discovery. The value of tRNA ligase as a therapeutic target would be enhanced if one knew more about the structural basis for catalysis and substrate specificity. An initial alanine scan of eight residues in the ligase domain showed that Lys114 in motif I, which is the site of covalent 
adenylylation (Xu et al. 1990), Glu266 and Gly267 in motif IV, and Lys284 and Lys286 in motif V are essential for Trl1 activity, whereas alanine substitutions at Asn116 (in motif I), Glu152, and Glu153 are benign (Sawaya et al. 2003). Here we conduct a more extensive structure-function analysis of the $\mathrm{N}$-terminal domain of Trl1, entailing alanine scanning and conservative substitutions at 46 positions. The results confirm an evolutionary connection between tRNA ligase and other covalent nucleotidyltransferases, but reveal a large number of essential components that are unique to the fungal tRNA ligase clade.

\section{RESULTS AND DISCUSSION}

\section{Velocity sedimentation of the ligase and kinase-CPD domains of Trl1}

Trl1 consists of an N-terminal adenylyltransferase/ligase domain, a central polynucleotide kinase domain, and a C-terminal CPD domain (Fig. 1). All three domains are essential in vivo, though they need not be linked covalently in the same polypeptide. For example, complementation of a lethal $\operatorname{trl} 1 \Delta$ mutation by the plasmid shuffle method can be achieved by expressing the ligase domain Trl1-(1-388) and the kinaseCPD domain Trl1-(389-827) as separate polypeptides (Sawaya et al. 2003). However, it is possible that the domains normally interact physically with one another, either in cis within a single Trl1 protomer or in trans to form a Trl1 homo-oligomer, in which case the complementation by separately expressed domains might entail physical interaction between the ligase and kinase-CPD proteins. Here we analyzed the native size of the ligase domain Trl1(1-388) and the kinase-CPD domain Trl1-(389-827) by zonal velocity sedimentation in a $15-30 \%$ glycerol gradient. Marker proteins catalase $(248 \mathrm{kDa})$, BSA $(66 \mathrm{kDa})$, and cytochrome $\mathrm{c}$ $(12 \mathrm{kDa})$ were included as internal standards in the gradient. After centrifugation, the polypeptide compositions of the odd-numbered gradient fractions were analyzed by SDS-PAGE. The ligase domain (calculated to be a $45-\mathrm{kDa}$ polypeptide) and the kinase-CPD domain (a 50-kDa polypeptide) both sedimented as a discrete peak overlapping the "light" side of the BSA peak (Fig. 2A). The adenylyltransferase activity profile of the ligase domain, gauged by reaction of the gradient fractions with $\left[\alpha^{32} \mathrm{P}\right]$ ATP to form a radiolabeled covalent ligase-AMP adduct, was coincident with the sedimentation profile of the Trl1-(1-388) protein (Fig. 2A). The polynucleotide kinase activity profile, assayed by label-transfer from $\left[\gamma^{32} \mathrm{P}\right]$ GTP to a $5^{\prime}$-OH 18-mer oligoribonucleotide, coincided with the abundance of the kinase-CPD polypeptide (Fig. 2B). These results are consistent with monomeric quaternary structures for the ligase and kinase-CPD domains. We tested interdomain complex formation by preincubating equal amounts of the ligase and kinase-CPD domains prior to glycerol gradient sedimentation. The sedimentation behavior of each component of the domain mixture was indistinguishable from what was seen when the domains were analyzed separately (not shown). That is, there was no evidence of formation of a heavier complex of the two proteins.

\section{Mutational analysis of the $\mathrm{N}$-terminal ligase domain}

An initial alanine scan of eight positions in the adenylyltransferase domain identified five amino acids (Lys114, Glu266,
A

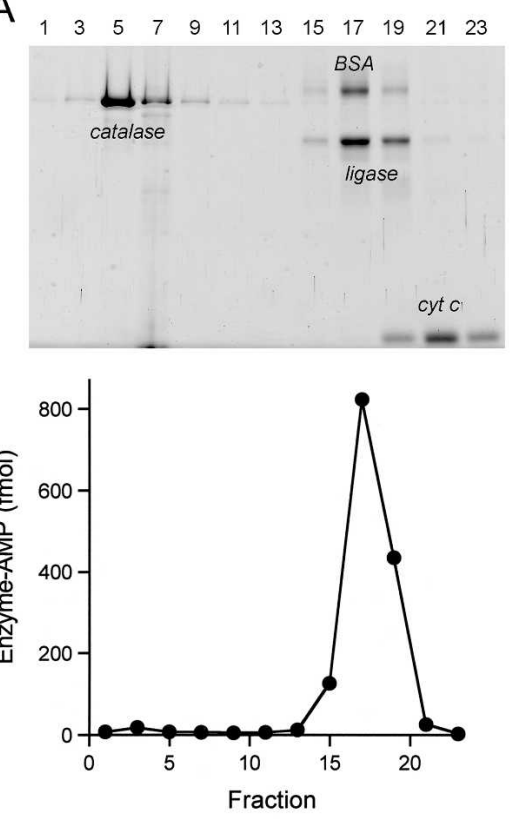

B
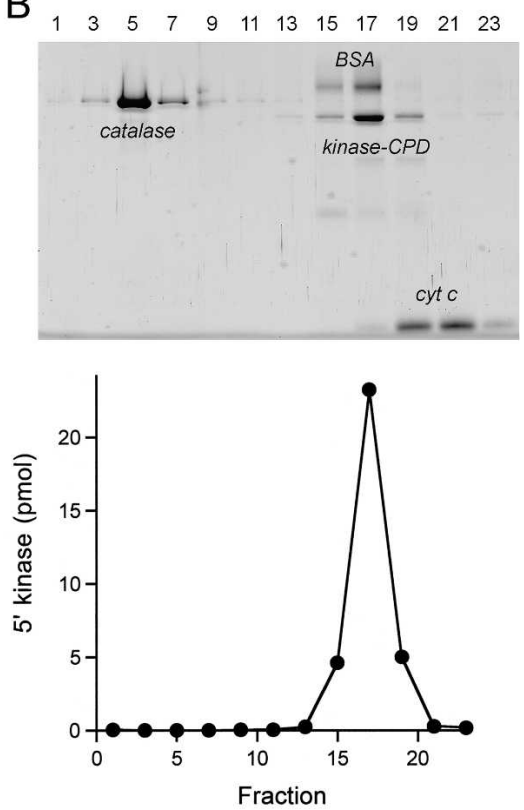

FIGURE 2. Sedimentation analysis of the ligase and kinase-CPD domains. Sedimentation of the ligase domain $(A)$ and kinase-CPD domain $(B)$ was performed as described in Materials and Methods. Aliquots $(20 \mu \mathrm{L})$ of the odd-numbered gradient fractions were analyzed by SDS-PAGE. The Coomassie blue-stained gel is shown. The positions of the recombinant Trl1 proteins and the internal standards catalase, BSA, and cytochrome $c$ are indicated. The adenylyltransferase and polynucleotide kinase activity profiles are shown below the polypeptide profiles. Adenylyltransferase reaction mixtures $(20 \mu \mathrm{L})$ containing $50 \mathrm{mM}$ Tris- $\mathrm{HCl}(\mathrm{pH} 8.0), 5 \mathrm{mM} \mathrm{MgCl} 2,2 \mathrm{mM}$ DTT, $20 \mu \mathrm{M}\left[\alpha-{ }^{32} \mathrm{P}\right] \mathrm{ATP}$, and $1 \mu \mathrm{L}$ of the indicated glycerol gradient fractions were incubated for $12 \mathrm{~min}$ at $37^{\circ} \mathrm{C}$. The products were analyzed by SDS-PAGE. The protein- $\left[{ }^{32} \mathrm{P}\right]$ AMP adduct was quantified by scanning the gel with a Fujix BAS2500 imaging apparatus. Polynucleotide kinase reaction mixtures $(10 \mu \mathrm{L})$ containing $70 \mathrm{mM}$ Tris- $\mathrm{HCl}(\mathrm{pH}$ 7.6), $5 \mathrm{mM}$ DTT, $10 \mathrm{mM}$ $\mathrm{MgCl}_{2}, 50$ pmol of 18-mer 5'-OH oligoribonucleotide r(AUUCCGAUAGUGACUACA), $40 \mu \mathrm{M}$ $\left[\gamma^{-32} \mathrm{P}\right] \mathrm{GTP}$, and $1 \mu \mathrm{L}$ of a 1:20 dilution of the indicated glycerol gradient fractions were incubated for $20 \mathrm{~min}$ at $37^{\circ} \mathrm{C}$. The products were analyzed by electrophoresis through a $15-\mathrm{cm}$ $20 \%$ polyacrylamide gel containing $7 \mathrm{M}$ urea in $45 \mathrm{mM}$ Tris-borate $1.25 \mathrm{mM}$ EDTA. The radiolabeled oligonucleotide products were quantified by scanning the gel. 
Gly267, Lys284, and Lys286) as essential for Trl1 activity (Sawaya et al. 2003); these essential residues are located within putative counterparts of nucleotidyltransferase motifs I $\left({ }^{114} \mathrm{KANG}^{117}\right)$, IV $\left({ }^{266} \mathrm{EGFVI}^{270}\right)$, and V $\left({ }^{282} \mathrm{FFKYK}^{286}\right)$. Here, we used the alignment of fungal tRNA ligases in Figure 1 to guide a more comprehensive mutational analysis of Trl1-(1-388). The strategy was to replace individual residues of interest with alanine and then test the TRL1-(1-388)Ala alleles for in vivo activity by complementation of S. cerevisiae $\operatorname{trl} 1 \Delta$. We selected 42 residues for alanine scanning. We focused on (1) conserved Arg, Lys, and His residues that we regarded as candidates for a direct role in catalysis of phosphoryl transfer or substrate binding; (2) conserved Glu and Asp residues as candidates for binding the requisite divalent cation cofactor(s); (3) conserved Gln, Asn, Ser, Thr, and Tyr side chains that might engage in hydrogen bonding with ATP or tRNA; and (4) conserved Phe residues that might engage in base-stacking interactions with ATP or tRNA.

TRL1-(1-388)-Ala alleles were cloned into a CEN TRP1 plasmid so as to place their expression under the control of the native TRL1 promoter. The plasmids were then cotransformed with a TRL1-(389-827) plasmid into a S. cerevisiae $\operatorname{trl} 1 \Delta$ strain. Growth of $\operatorname{trl} \Delta$ is contingent on maintenance of a wild-type TRL1 allele on a CEN URA3 plasmid (Sawaya et al. 2003). Therefore, the $\operatorname{trl} \Delta$ strain is unable to grow on agar medium containing 5-FOA (5-fluoroorotic acid, a drug which selects against the URA3 plasmid) unless it is first transformed with genes encoding biologically active ligase and kinase-CPD enzymes.

Twenty-three of the TRL1-(1-388)-Ala transformants failed to give rise to 5-FOA-resistant colonies after $7 \mathrm{~d}$ of incubation at $18^{\circ} \mathrm{C}, 25^{\circ} \mathrm{C}$, or $30^{\circ} \mathrm{C}$; thus these 23 alanine mutations were deemed lethal in vivo. The new residues defined as essential by the alanine scan were Arg66, Arg82, Lys86, Phe87, Phe88, Ser134, Lys135, His136, Ser137, His148, Glu184, Asp187, Asp188, Glu191, Glu192, His193, His207, Asn210, Arg271, Arg298, Glu299, Lys302, and Arg352. The essential amino acids are denoted by $\mid$ over the aligned protein sequences in Figure 1. Failure of in vivo complementation could result from loss of catalytic function of the mutated proteins or mutational effects on intracellular Trl1-(1-388) protein concentration (via decreased synthesis or accelerated turnover) or localization. Lacking an antibody to Trl1, we did not determine the steady-state levels of the mutant proteins. Thus, we cannot assign the basis for in vivo lethality for every defective mutant. However, an analysis of the catalytic activity of recombinant versions of selected mutant Trl1-(1-388) proteins was informative (see below).

Nineteen other TRL1-(1-388)-Ala mutants supported colony formation during selection on 5 -FOA at either $25^{\circ} \mathrm{C}$ or $30^{\circ} \mathrm{C}$. The viable TRL1-(1-388)-Ala strains were tested for growth on rich medium (YPD agar) at $18^{\circ} \mathrm{C}, 25^{\circ} \mathrm{C}, 30^{\circ} \mathrm{C}$, and $37^{\circ} \mathrm{C}$. Two of the mutants, $N 212 A$ and $F 216 A$, displayed a temperature-sensitive phenotype, whereby $N 212 A$ and
F216A cells grew as well as "wild-type" TRL1-(1-388) TRL1-(389-827) cells at $18^{\circ} \mathrm{C}, 25^{\circ} \mathrm{C}$, and $30^{\circ} \mathrm{C}$, but failed to grow at $37^{\circ} \mathrm{C}$ (scored as $t$ in Table 2 and denoted by $\Delta$ in Fig. 1). Seventeen other TRL1-(1-388)-Ala strains grew at all temperatures and their colony sizes were similar to that of wild-type TRL1-(1-388) TRL1-(389-827) cells (Tables 1 and 2). We surmise that Asp85, Asn89, Glu92, Thr96, Asp110, Thr212, Cys133, Thr180, Asn242, Glu289, Arg295, Gln296, Thr301, Lys313, Arg315, Lys316, and Thr321 are not essential for tRNA ligase activity in vivo. The nonessential residues are denoted by + in Figure 1 .

We tested the effects of conservative substitutions at the 23 positions defined as essential by the present alanine scan and at the four essential nonglycine residues identified previously. Arginine was replaced by lysine and glutamine, lysine by arginine and glutamine, histidine by asparagine and glutamine, glutamate by aspartate and glutamine, aspartate by glutamate and asparagine, asparagine by aspartate and glutamine, serine by threonine, and phenylalanine by leucine. A total of 50 conservative mutants were tested by plasmid shuffle for $\operatorname{trl} 1 \Delta$ complementation; the results are shown in Tables 1 and 2 .

\section{Structure-activity relationships at essential residues of motifs la, IV, and V}

Four of the essential residues are located within or immediately adjacent to nucleotidyltransferase motifs Ia, IV, and V. Crystal structures of exemplary nucleotidyltransferase family members show that motif Ia is located atop a $\beta$-hairpin loop on the surface of the enzyme; the Arg or Lys chain of the Ser-(Arg/Lys) dipeptide is proposed to contact the $\gamma$ phosphate of the NTP substrate and/or the reactive $5^{\prime}-\mathrm{PO}_{4}$ end of the polynucleotide to be sealed (Håkansson et al. 1997; Odell et al. 2000; Fabrega et al. 2003; Deng et al. 2004; Ho et al. 2004; Pascal et al. 2004). Mutations of the motif Ia Lys/Arg residue resulted in loss of activity in the case of T4 Rnl1, T4 Rnl2, Chlorella virus DNA ligase, and mammalian RNA capping enzyme (Odell et al. 2000; Sawaya and Shuman 2003; Wang et al. 2003; Yin et al. 2003). Here we found that Lys135 in motif Ia of Trl1 was strictly essential, i.e., Arg and Gln substitutions were lethal (Table 1). The two flanking serine residues of motif Ia, Ser134 and Ser137, were also strictly essential-i.e., threonine substitutions were lethal. Thus, the hydroxyl group is critical at positions 134 and 137 and the extra bulk of the threonine methyl group is deleterious. Replacing the essential His 136 side chain of motif Ia with glutamine was lethal, but the asparagine substitution restored Trl1 function in vivo. These findings argue against a role for His136 as a general acid-base catalyst; rather they suggest that hydrogen bonding of the histidine $\mathrm{N} \delta$ atom (on which the $\mathrm{N} \delta$ of Asn can superimpose) is the relevant functional contribution of His 136.

The motif IV carboxylate side chain is essential for all members of the covalent nucleotidyltransferase superfamily (Sriskanda and Shuman 2002a; Sawaya and Shuman 2003; 
TABLE 1. Effect of missense mutations on Trl1 ligase activity in vivo

\begin{tabular}{|c|c|}
\hline Trl1 (1-388) mutation & trl18 complementation \\
\hline R66A & lethal \\
\hline R66K & lethal \\
\hline R66Q & lethal \\
\hline R82A & lethal \\
\hline R82K & lethal \\
\hline R82Q & lethal \\
\hline D85A & +++ \\
\hline K86A & lethal \\
\hline K86R & lethal \\
\hline K86Q & lethal \\
\hline F87A & lethal \\
\hline F87L & lethal \\
\hline F88A & lethal \\
\hline F88L & lethal \\
\hline N89A & +++ \\
\hline E92A & +++ \\
\hline T96А & +++ \\
\hline D110A & +++ \\
\hline $\mathrm{T} 112 \mathrm{~A}$ & +++ \\
\hline K114R & lethal \\
\hline K114Q & lethal \\
\hline C133A & +++ \\
\hline S134A & lethal \\
\hline $\mathrm{S} 134 \mathrm{~T}$ & lethal \\
\hline K135A & lethal \\
\hline K135R & lethal \\
\hline K135Q & lethal \\
\hline $\mathrm{H} 136 \mathrm{~A}$ & lethal \\
\hline $\mathrm{H} 136 \mathrm{~N}$ & +++ \\
\hline H136Q & lethal \\
\hline S137A & lethal \\
\hline $\mathrm{S} 137 \mathrm{~T}$ & lethal \\
\hline $\mathrm{H} 148 \mathrm{~A}$ & lethal \\
\hline $\mathrm{H} 148 \mathrm{~N}$ & lethal \\
\hline H148Q & lethal \\
\hline T180A & +++ \\
\hline E184A & lethal \\
\hline E184D & lethal \\
\hline E184Q & lethal \\
\hline D187A & lethal \\
\hline D187N & +++ \\
\hline D187E & lethal \\
\hline D188A & lethal \\
\hline D188N & lethal \\
\hline D188E & lethal \\
\hline E191A & lethal \\
\hline E191Q & lethal \\
\hline E191D & lethal \\
\hline E192A & lethal \\
\hline E192Q & +++ \\
\hline E192D & lethal \\
\hline H193A & lethal \\
\hline $\mathrm{H} 193 \mathrm{~N}$ & lethal \\
\hline H193Q & lethal \\
\hline $\mathrm{H} 207 \mathrm{~A}$ & lethal \\
\hline $\mathrm{H} 207 \mathrm{~N}$ & lethal \\
\hline H207Q & lethal \\
\hline
\end{tabular}

$(+++)$ Wild-type growth
Wang et al. 2003; Yin et al. 2003). Here we found that replacing the motif IV Glu266 side chain of Trl1 by either glutamine or aspartate resulted in loss of function. We surmise that a carboxylate moiety is essential in Trl1 and that activity depends on the proper distance of the carboxylate from the main chain. The motif IV carboxylate coordinates a divalent cation in the crystal structures of DNA ligases (Odell et al. 2000; Pascal et al. 2004); we invoke a similar role for Glu266 in Trl1. The Arg271 side chain immediately flanking motif IV can be functionally replaced by lysine, but not glutamine (Table 2). Many, but not all, nucleotidyltransferases have an arginine or lysine at the position corresponding to Arg271 of Trll. The equivalent residue is a lysine in T4 Rnl2; mutation of this lysine to Ala abolishes Rnl2 activity (Yin et al. 2003). Exemplary crystal structures show that the motif IV Arg/Lys side chain comprises half of an ion pair that stabilizes the architecture of the active site (Odell et al. 2000; Deng et al. 2004; Ho et al. 2004).

The two motif $\mathrm{V}$ lysines are critical for the function of ATP-dependent DNA and RNA ligases and GTP-dependent mRNA capping enzymes (Sriskanda and Shuman 2002b; Sawaya and Shuman 2003; Yin et al. 2003). Here we find that neither arginine nor glutamine can function in lieu of Lys284 and Lys286 of Trl1 (Table 2). The motif V lysines contact the phosphates of the NTP substrate, the covalent ligase-adenylate intermediate, or the polynucleotide-adenylate intermediate in various crystal structures of nucleotidyltransferase superfamily members (Subramanya et al. 1996; Håkansson et al. 1997; Odell et al. 2000; Pascal et al. 2004).

\section{An essential Rnl1-like module located proximal to motif I}

Five of the essential side chains of Trl1 mapped to a bipartitite module upstream of the AMP attachment site, of consensus sequence $\mathrm{RGLF}(\mathrm{X})_{9-17}{ }_{17} \mathrm{RGYDKFFN}$ (essential residues underlined), that is conserved in bacteriophage T4 Rnll and the Rnl1-like ligases encoded by other phages and by certain baculoviruses (Wang et al. 2003; Martins and Shuman 2004; Blondal et al. 2005), but not in other branches of the covalent nucleotidyltransferase superfamily. Mutational analysis of T4 Rnl1 showed that the arginine of the RGLF peptide and the lysine and second phenylalanine of the KFFN peptide were essential for RNA strand joining in vitro (Wang et al. 2003). Here we find that Arg66 and Arg82 are strictly essential for Trll function; lysine or glutamine substitutions were lethal at both positions (Table 1). Lys86 is also strictly essential, insofar as arginine or glutamine substitutions were lethal (Table 1). We surmise that the aromaticity of Phe87 and Phe88 are essential, because leucine substitutions were lethal at either position (Table 1).

\section{A candidate motif III counterpart in TRNA ligase}

Analysis of Trl1 primary structure did not reveal any obvious counterparts of nucleotidyltransferase motifs III or IIIa found 
TABLE 2. Effect of missense mutations on Trl1 ligase activity in vivo Trl1 (1-388) mutation trl1 complementation

\begin{tabular}{ll}
\hline N210A & lethal \\
N210D & ts \\
N210Q & ts \\
N212A & ts \\
F216A & ts \\
N242A & +++ \\
E266Q & lethal \\
E266D & lethal \\
R271A & lethal \\
R271K & +++ \\
R271Q & lethal \\
K284R & lethal \\
K284Q & lethal \\
K286R & lethal \\
K286Q & lethal \\
E289A & +++ \\
R295A & +++ \\
Q296A & +++ \\
R298A & +++ \\
R298K & lethal \\
R298Q & lethal \\
E299A & lethal \\
E299Q & lethal \\
E299D & cs \\
T301A & lethal \\
K302A & +++ \\
K302R & lethal \\
K302Q & lethal \\
K313A & lethal \\
R315A & +++ \\
K316A & +++ \\
T321A & +++ \\
R352A & +++ \\
R352K & lethal \\
R352Q & lethal \\
\hline & lethal \\
\hline$+r$ A &
\end{tabular}

$(+++)$ Wild-type growth

(ts) Temperature-sensitive

(cs) Cold-sensitive

in other branches of the ligase/capping enzyme superfamily. The consensus motif III sequence is $\phi \phi \phi D G E \phi \phi$ (where $\phi$ is an aliphatic side chain). In our initial small-scale alanine scan of Trl1, we targeted the ${ }^{152} \mathrm{Gly}-\mathrm{Glu}^{153}$ dipeptide as a possible counterpart of motif III, but found that Trl1 activity in vivo was unaffected by the G152A or E153A mutations (Sawaya et al. 2003). This raises two possibilities: either (1) Trl1 has no counterpart of motif III or (2) it has a counterpart of motif III, but its sequence has diverged from the consensus. We noted previously that the aspartate preceding the glycine, though essential when present in ligases or capping enzymes, is not strictly conserved in all nucleotidyltransferases (Wang et al. 1997). Also, the glycine is replaced in some ligases and capping enzymes by alanine, serine, cysteine, or threonine. The glutamate is the only strictly conserved motif III residue, and it is essential for ligase or capping enzyme activity in all cases tested (Sriskanda and Shuman 2002a; Sawaya and Shuman 2003; Zhu and Shuman 2005). The present mutational analysis of Trl1 identified three essential glutamate side chains in the segment between motif Ia and motif IV. Of these, Glu184 is located within the sequence ${ }^{179}$ VTAVAEYC ${ }^{186}$, which we propose is the motif III equivalent of Trl1 (Fig. 1). We find that Glu184 is strictly essential, because conservative substitutions with glutamine or aspartate were lethal in vivo (Table 1). The motif III glutamate contacts the ribose sugar of the NTP substrate, the covalent enzyme-NMP intermediate, or the polynucleotide-adenylate intermediate in various crystal structures of nucleotidyltransferase superfamily members (Subramanya et al. 1996; Håkansson et al. 1997; Pascal et al. 2004). We propose a similar role for Glu184 in Trl1.

\section{Essential structural elements unique to tRNA ligases}

The essential His148 side chain is conserved in all fungal tRNA ligases and is located between motifs Ia and III. Replacing His 148 with either asparagine or glutamine was lethal in vivo (Table 1). These conservative replacements are mimetics of the hydrogen-binding capacity of the $\mathrm{N} \delta$ and $\mathrm{N} \epsilon$ atoms, respectively. Conceivably, the His149 side chain either (1) makes bivalent polar interactions via $\mathrm{N} \delta$ and $\mathrm{N} \epsilon$, (2) makes ionic interactions as the protonated form, (3) serves as a general acid or general base catalyst during one or more steps of the ligation reaction, or (4) depends on its aromatic character for sustaining Trl1 function. It is noteworthy that Sidrauski et al. (1996) identified a viable mutant allele of TRL1 with a single $\mathrm{H} 148 \mathrm{Y}$ coding change that selectively impaired the function of Trl1 in HAC1 mRNA splicing during the unfolded protein response without affecting tRNA splicing in vivo. The ability of tyrosine to function in lieu of His149 excludes options 1 and 2 above and weighs against a mechanism whereby this residue acts as an essential general base catalyst.

Seven essential side chains are located within the region downstream of motif III, five of which (Asp187, Asp188, Glu191, Glu192, and His193) are clustered within an acidic patch. Based on the crystal structures of other ligases, we would predict that this patch projects on the surface of the nucleotidyltransferase domain. Whereas Asp188 and Glu191 are strictly essential, the ligase retained function when either Asp187 or Glu192 were replaced by their isosteric amide derivatives (Table 1). We surmise that hydrogen bonding capacity is the relevant property of the Asp187 and Glu192 side chains. Neither His193 nor His207 tolerated replacement by asparagine or glutamine. The ligase domain was functional in vivo when Asn 210 was replaced by either aspartate or glutamine. The $N 210 D$ and $N 210 Q$ strains grew at $25^{\circ} \mathrm{C}$ and $30^{\circ} \mathrm{C}$, but not at $37^{\circ} \mathrm{C}$ (scored as $t$ in Table 2).

Five essential residues mapped to the ligase segment downstream of motif V. Changing Arg298 to lysine or glutamine 
was lethal in vivo, suggesting that this residue makes an essential bidentate interaction. Replacing Lys302 with either arginine or glutamine was lethal as well. Whereas changing Glu299 to aspartate was lethal, the glutamine mutant grew at $25^{\circ} \mathrm{C}, 30^{\circ} \mathrm{C}$, and $37^{\circ} \mathrm{C}$, but not at $18^{\circ} \mathrm{C}$ (scored as cs is Table 2). The most distal essential residue, Arg352, also could not be replaced by Lys or Gln. The necessity of Arg352 explains the previous finding that deletion of the segment from amino acids 321-362 abolished ligase function in vivo (Sawaya et al. 2003).

\section{Effects of selected mutations on adenylyltransferase activity in vitro}

Recombinant Trl1-(1-388) proteins containing lethal conservative substitutions in motifs I, IV, and V were produced in Escherichia coli as $\mathrm{His}_{10}$-tagged fusions and purified from soluble bacterial extracts by $\mathrm{Ni}$-agarose chromatography in parallel with wild-type Trl1-(1-388) (Fig. 3, top panel). The adenylyltransferase activity of wild-type Trl1-(1-388) was evinced by label transfer from $\left[\alpha-{ }^{32} \mathrm{P}\right]$ ATP to form a covalent enzyme- $\left[{ }^{32} \mathrm{P}\right]$ adenylate adduct detectable by SDS-PAGE and autoradiography (Fig. 3, bottom panel). The yield of Trl1-(1-388)-[ $\left.{ }^{32} \mathrm{P}\right]$ AMP is proportional to input enzyme (Sawaya et al. 2003). Changing the motif I lysine (Lys114) to either arginine or glutamine abolished

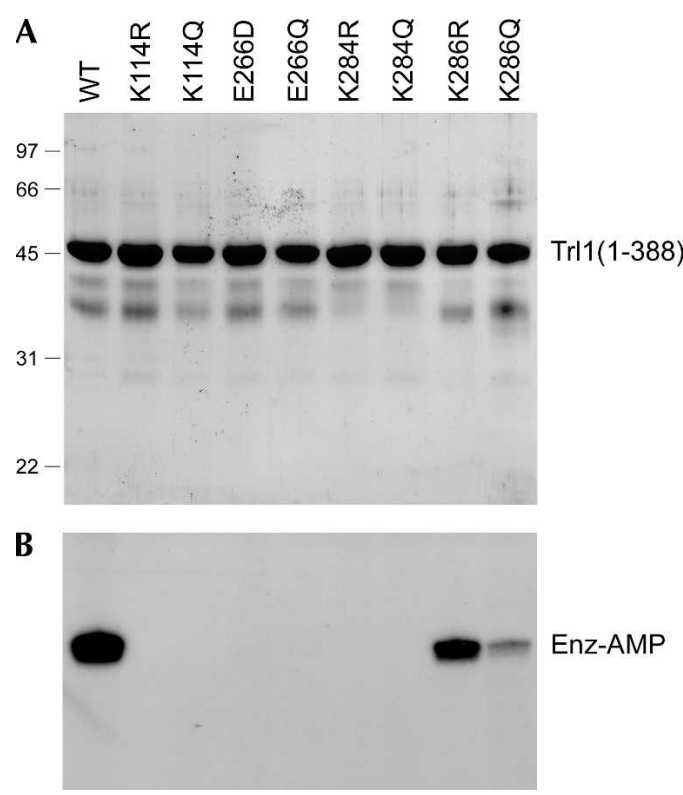

FIGURE 3. Mutational effects on adenylyltransferase activity in vitro. (A) Aliquots $(8 \mu \mathrm{g})$ of recombinant wild-type and mutated Trl1(1-388) proteins were analyzed by SDS-PAGE. The Coomassie bluestained gel is shown. The positions and sizes (in kilodaltons) of marker polypeptides are indicated on the left. (B) Adenylyltransferase reaction mixtures $(20 \mu \mathrm{L})$ containing $50 \mathrm{mM}$ Tris- $\mathrm{HCl}(\mathrm{pH} 8.0), 5 \mathrm{mM} \mathrm{MgCl}_{2}$, $2 \mathrm{mM}$ DTT, $20 \mu \mathrm{M}\left[\alpha-{ }^{32} \mathrm{P}\right] \mathrm{ATP}$, and $1 \mu \mathrm{g}$ of the indicated ligase protein were incubated for $12 \mathrm{~min}$ at $37^{\circ} \mathrm{C}$. The products were resolved by SDS-PAGE and visualized by autoradiography.
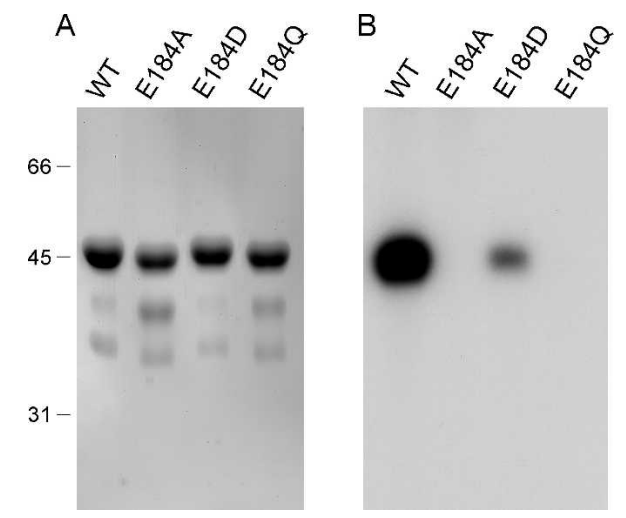

FIGURE 4. Effects of motif III glutamate mutations on adenylyltransferase activity. $(A)$ Aliquots $(8 \mu \mathrm{g})$ of recombinant wild-type Trl1(1-388) and mutant proteins E184A, E184D, and E184Q were analyzed by SDS-PAGE. The Coomassie blue-stained gel is shown. The positions and sizes (in kilodaltons) of marker polypeptides are indicated on the left. (B) Adenylyltransferase reaction mixtures $(20 \mu \mathrm{L})$ contained $50 \mathrm{mM}$ Tris- $\mathrm{HCl}(\mathrm{pH} 8.0), 5 \mathrm{mM} \mathrm{MgCl}_{2}, 2 \mathrm{mM}$ DTT, $20 \mu \mathrm{M}\left[\alpha-{ }^{32} \mathrm{P}\right] \mathrm{ATP}$, and $1 \mu \mathrm{g}$ of the indicated ligase protein. The products were resolved by SDSPAGE and visualized by autoradiography.

adenylyltransferase activity. Thus, arginine is not a suitable nucleophile for attack by Trll on the $\alpha$ phosphate of ATP. Replacing the motif IV glutamate (Glu266) with either aspartate or glutamine also abolished ligase adenylylation; this finding is consistent with the proposed metal-binding function of Glu266. Conservative mutations of motif V Lys284 eliminated adenylyltransferase activity as well. Thus, the in vivo lethality of these mutations is readily explained by inability to perform the first chemical step of the three-step RNA ligation reaction. In contrast, the motif V K286R mutant retained adenylyltransferase activity (the yield of K286R-AMP complex being $21 \%$ of the wild-type level), suggesting that lethality in vivo is caused by a defect downstream of step 1 of the ligation pathway. The K286Q mutant was $5 \%$ as active as wild-type ligase in the autoadenylylation reaction (Fig. 3). We also purified mutants of the putative motif III glutamate, E184A, E184D, and E184Q (Fig. 4A). The E184A and E184Q mutations abolished adenylyltransferase activity; the E184D mutant was 13\% as active as wild-type Trl1-(1-388) (Fig. 4B).

\section{Candidate trifunctional tRNA ligases from Leishmania and Trypanosoma}

The phylogenetic distribution of Trl1-like proteins is surprisingly narrow, given the wide occurrence of tRNA introns in archaea and eukarya. Trll homologs are found in all genera of fungi for which genome sequences are available. Trl1-like proteins are absent from the proteomes of archaea and metazoans. This may be because archaea and metazoans use a different end-joining mechanism for tRNA splicing than do fungi (Filipowicz and Shatkin 1983; Laski et al. 1983; Zofallova et al. 2000). None of the specific 
proteins responsible for tRNA ligation in archaea or metazoans have been identified nor have their genes been cloned as yet. This scenario, in which the Trll-like pathway in metazoans is either redundant or performed by enzymes without recognizable structural similarity to fungal tRNA ligases, recommends Trll as an excellent target for antifungal drug discovery.

One dividend of assembling a comprehensive structurefunction map of Trl1 is that it improves our ability to confidently identify homologous tRNA splicing enzymes from novel sources, by focusing on conservation of the amino acids shown to be essential, even where global primary structure similarity is not high. This approach proved extremely fruitful in the case of another essential fungal RNA processing enzyme, the RNA triphosphatase component of the mRNA capping apparatus (Shuman 2002). Fungi and metazoans diverge completely with respect to the structure and mechanism of their RNA triphosphatase enzymes. The fungal triphosphatases belong to a superfamily of metal-dependent phosphohydrolases that includes the RNA triphosphatases of many nonfungal unicellular eukarya, including Plasmodium, Trypanosoma, and Giardia. A cap-centric scheme of eukaryotic phylogeny suggests a common ancestry for the mRNA processing machinery of fungi and protozoa (Shuman 2002).

To see whether this relationship extends to tRNA splicing, we searched for Trll homologs encoded by protozoan organisms and thereby identified candidate tRNA ligases from the kinetoplastid protozoa Trypanosoma brucei, Trypanosoma cruzi, and Leishmania major (Fig. 5). The 870-amino-acid T. brucei protein (designated TbrTrl1) is composed of an $\mathrm{N}$-terminal adenylyltransferase/ligase domain, a central kinase module, and a C-terminal CPD module, each of which resembles the equivalent functional domain of yeast Trll. The ligase domain of TbrTrll contains putative counterparts of motfis I, Ia, III, IV, and V (shaded in Fig. 5), as well as a T4 Rnl1-like segment upstream of the predicted AMP attachment site. Eighteen individual amino acids found in the protozoan ligase domains that we predict correspond to essential residues of Trl1 identified in the present study are indicated by dots over the sequences in Figure 5. A Blast search using the ligase domain of TbrTrll readily identified the fungal tRNA ligases as the closest homologs, followed by bacteriophage Rnll. The central kinase domain of the protozoan tRNA ligases contains the GxGKT and RxxxR motifs found in yeast Trl1 and T4 polynucleotide kinase (Wang et al. 2002; Sawaya et al. 2003). The C-terminal CPD motif of the protozoan proteins contains the $\mathrm{H} \phi \mathrm{T} \phi$ motifs of the $2 \mathrm{H}$ phosphotransferase enzymes that are present in fungal tRNA ligases (Mazumder et al. 2002; Sawaya et al. 2003). We found no credible homologs of the protozoan proteins in metazoan proteomes. Given that Trypanosoma and Leishmania are significant human pathogens, we regard the family of Trl1-like ligases as targets for the discovery of new broad-spectrum antifungal and antiprotozoal drugs.

Finally, as we were preparing this study for submission, Englert and Beier (2005) reported the identification of

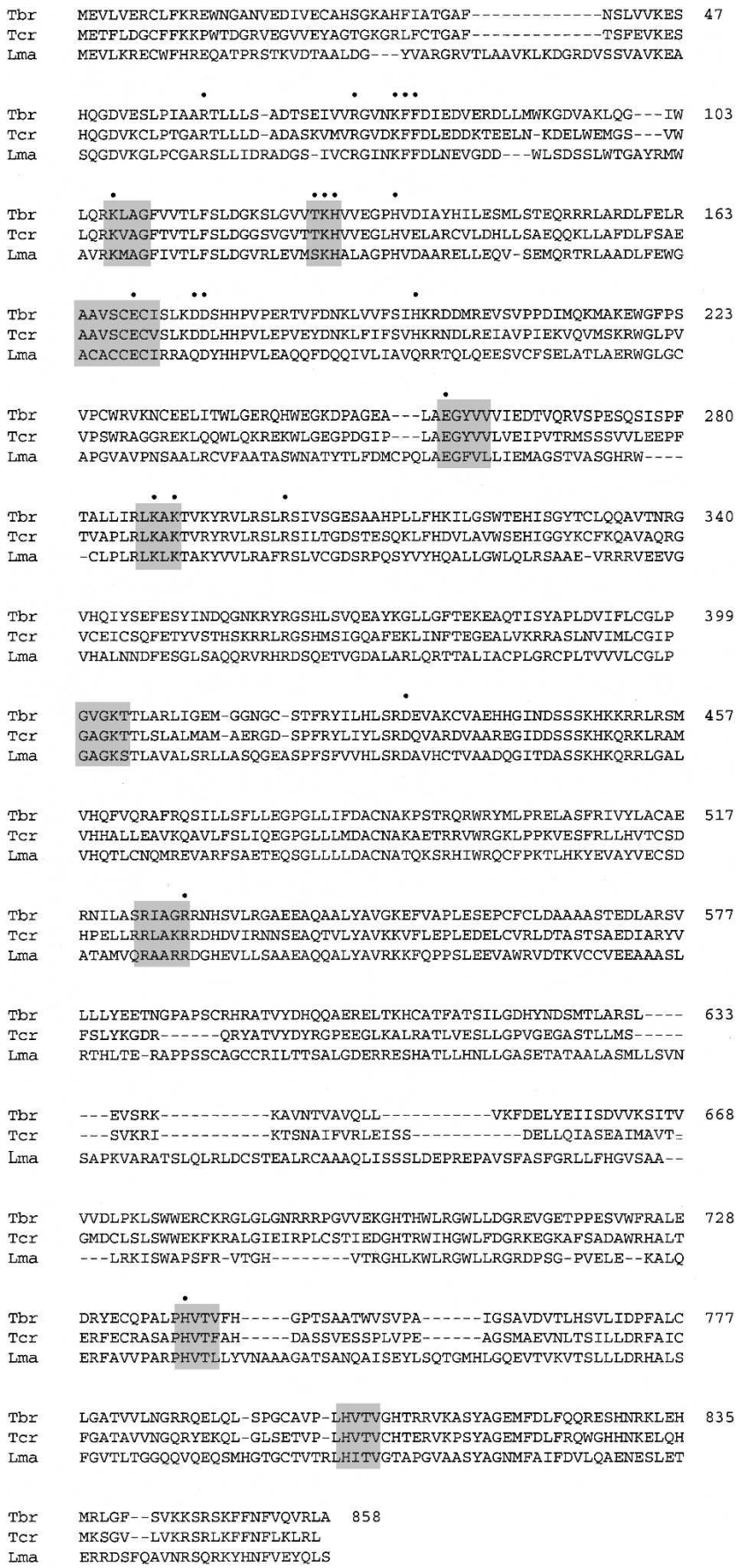

FIGURE 5. Trl1-like tRNA ligases from Trypanosoma and Leishmania. The amino acid sequence of a Trl1 homolog from T. brucei (Sanger Institute $T b 10.6 \mathrm{k} 15.2400$ ) is aligned to related proteins encoded by T. cruzi (TIGR_5693) and L. major (Sanger Institute LM36.1.Contig1). Nucleotidyl transferase motifs I, Ia, III, IV, and V, kinase motifs GxGK(S/T) and RxxxR, and $2 \mathrm{H}$ motifs HVTV in the CPD domain are highlighted in shaded boxes. Individual amino acids in the protozoan proteins corresponding to known essential residues of yeast Trll are indicated by $\bullet$ above the alignment.

the cDNA encoding tRNA ligase from the plant Arabidopsis thaliana. Although plant RNA ligases catalyze the same set of healing and sealing reactions as does Trll (Gegenheimer et al. 
1983; Schwartz et al. 1983; Pick and Hurwitz 1986; Pick et al. 1986), the plant tRNA ligase polypeptide has scant similarity to fungal Trl1 (Englert and Beier 2005). It will be of interest to test whether the protozoan and plant ligases can function in yeast in lieu of Trl1.

\section{MATERIALS AND METHODS}

\section{Yeast vectors encoding missense mutants of Trl1-(1-388)}

Missense mutations and overlapping diagnostic restriction sites were introduced into the TRL1-(1-388) open reading frame (ORF) via the two-stage PCR overlap extension method as described previously (Sawaya et al. 2003). The mutated PCR products were digested with BamHI and SacII and inserted into the isolated vector backbone of BamHI/SacII-cut CEN TRP1 plasmid pSE358-TRL1(1-388) (Sawaya et al. 2003). The mutant gene thereby replaces with wildtype ORF; expression of TRL1-(1-388) in this vector is under the control of the native TRL1 promoter. The inserts were sequenced completely to confirm the presence of the desired mutations and to exclude the acquisition of unwanted coding changes during PCR amplification and cloning.

\section{Test of Trl1-(1-388) function by plasmid shuffle}

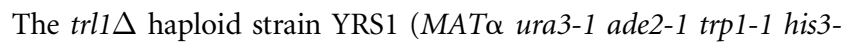
11,15 leu2-3,11 can1-100 trl1::kanMX p360-TRL1) was cotransformed with a CEN TRP1 plasmid bearing a wild-type or mutated version of TRL1-(1-388) and a CEN ADE2 TRL1-(389-827) plasmid (Sawaya et al. 2003). Transformants were selected on medium lacking tryptophan and adenine. Two individual colonies were transferred to fresh selective medium. The isolates were then streaked on agar medium containing $0.75 \mathrm{mg} / \mathrm{mL} 5$-FOA. The plates were incubated at $18^{\circ} \mathrm{C}, 25^{\circ} \mathrm{C}$, and $30^{\circ} \mathrm{C}$. Lethal mutations were those that did not allow formation of FOA-resistant colonies after $7 \mathrm{~d}$ at any of the temperatures tested. Other mutated alleles supported FOA-resistant colony formation at one or more of the growth temperatures. At least two individual colonies from each streak were picked from the FOA plate, transferred to yeast extract/peptone/dextrose (YPD) medium and then tested for growth on YPD agar at $18^{\circ} \mathrm{C}, 25^{\circ} \mathrm{C}$, $30^{\circ} \mathrm{C}$ and $37^{\circ} \mathrm{C}$. TRL1-(1-388) mutants that formed "wild-type" sized colonies at all temperatures were scored as +++ . Temperature sensitive $(t s)$ mutants grew at all temperatures except $37^{\circ} \mathrm{C}$. Coldsensitve (cs) mutants grew at all temperatures except $18^{\circ} \mathrm{C}$.

\section{Plasmids for expression of Trl1-(1-388) in bacteria}

The T7-based expression vector pET28a-His ${ }_{10}$ was constructed by replacing the MluI-NdeI fragment of pET28a (encoding a $\mathrm{His}_{6}$ tag) with the MluI-NdeI fragment of pET16b (encoding a His $\mathrm{H}_{10}$ tag). The ORF encoding the ligase domain Trl1-(1-388) was excised from pUC-Trl1-(1-388) with BamHI and SacI and inserted into pET28a-His $_{10}$ to yield pET28a-His 10 Trl1-(1-388). DNA fragments encoding mutated versions of the ligase domain mutants were excised from the respective yeast p358-TRL1-(1-388) plasmids with BamHI and SacII and inserted into the isolated vector backbone of BamHI/SacII-cut pET28a-His ${ }_{10} \operatorname{Trl1}-(1-388)$ in lieu of the wild-type ORF.

\section{Recombinant Trl1-(1-388) proteins}

pET28a-His ${ }_{10}$ Trl1-(1-388) plasmids were transformed into E. coli BL21(DE3). Single kanamycin-resistant colonies were inoculated into Luria-Bertani medium containing $0.06 \mathrm{mg} / \mathrm{mL}$ kanamycin. One-hundred-milliliter cultures were incubated at $37^{\circ} \mathrm{C}$ until the $A_{600}$ reached 0.5 . The cultures were placed on ice for $30 \mathrm{~min}$, adjusted to $0.3 \mathrm{mM}$ isopropyl- $\beta$-D-thiogalactopyroanoside (IPTG) and $2 \%(\mathrm{v} / \mathrm{v})$ ethanol. Incubation was continued at $17^{\circ} \mathrm{C}$ for $15 \mathrm{~h}$ with constant shaking. Cells were harvested by centrifugation and the pellets stored at $-80^{\circ} \mathrm{C}$. All subsequent procedures were performed at $4^{\circ} \mathrm{C}$. Thawed bacterial pellets were resuspended in $7 \mathrm{~mL}$ of lysis buffer $(50 \mathrm{mM}$ Tris- $\mathrm{HCl}$ at $\mathrm{pH} 7.5,1.2 \mathrm{M} \mathrm{NaCl}$, $15 \mathrm{mM}$ imidazole, $10 \%$ glycerol, $1 \mathrm{mM}$ benzamidine, $0.2 \mathrm{mM}$ phenylmethylsulfonyl fluoride) containing $1 \mathrm{mg} / \mathrm{mL}$ lysozyme and $0.2 \%$ Triton $\mathrm{X}-100$. The lysates were sonicated to reduce viscosity. Insoluble material was removed by centrifugation in a Sorvall SS34 rotor at $14,000 \mathrm{rpm}$ for $45 \mathrm{~min}$. The soluble extracts were applied to $0.75-\mathrm{mL}$ columns of Ni-NTA agarose (Qiagen) equilibrated with lysis buffer. The columns were washed with $5 \mathrm{~mL}$ of lysis buffer and then eluted step-wise with buffer A (50 mM Tris$\mathrm{HCl}$ at $\mathrm{pH} 7.5,0.2 \mathrm{M} \mathrm{NaCl}, 10 \%$ glycerol) containing 100 and $300 \mathrm{mM}$ imidazole. The polypeptide compositions of the column fractions were monitored by SDS-polyacrylamide gel electrophoresis (PAGE). The recombinant Trl1-(1-388) proteins were retained on the column and recovered in the $300 \mathrm{mM}$ imidazole eluates. Peak fractions were pooled and stored at $-80^{\circ} \mathrm{C}$. Protein concentrations were determined using the BioRad dye-binding assay with bovine serum albumin as a standard.

\section{Velocity sedimentation}

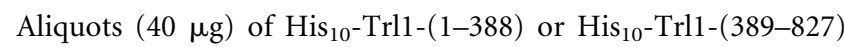
were mixed with catalase $(30 \mu \mathrm{g})$, BSA $(30 \mu \mathrm{g})$, and cytochrome c $(30 \mu \mathrm{g})$. The protein mixture was applied to a $4.8-\mathrm{mL} 15-30 \%$ glycerol gradient containing $50 \mathrm{mM}$ Tris- $\mathrm{HCl}$ ( $\mathrm{pH} 8.0), 200 \mathrm{mM}$ $\mathrm{NaCl}, 1 \mathrm{mM}$ EDTA, $2.5 \mathrm{mM}$ DTT, and $0.1 \%$ Triton X-100. The gradient was centrifuged in a Beckman SW50 rotor at 50,000 rpm for $16 \mathrm{~h}$ at $4^{\circ} \mathrm{C}$. Fractions $(\sim 0.2 \mathrm{~mL})$ were collected from the bottom of the tubes.

\section{ACKNOWLEDGMENTS}

This research was supported by NIH grant GM42498. S.S. is an American Cancer Society Research Professor.

Received January 27, 2005; accepted March 2, 2005.

\section{REFERENCES}

Abelson, J., Trotta, C.R., and Li, H. 1998. tRNA splicing. J. Biol. Chem. 273: 12685-12688.

Amitsur, M., Levitz, R., and Kaufman, G. 1987. Bacteriophage T4 anticodon nuclease, polynucleotide kinase, and RNA ligase reprocess the host lysine tRNA. EMBO J. 6: 2499-2503. 
Apostol, B.L., Westaway, S.K., Abelson, J., and Greer, C.L. 1991. Deletion analysis of a multifunctional yeast tRNA ligase polypeptide: Identification of essential and dispensable functional domains. J. Biol. Chem. 266: 7445-7455.

Blondal, T., Thorisdottir, A., Unnsteinsdottir, U., Hjorleifsdottir, S. Aevarsson, A., Ernstsson, S., Fridjonsson, O.H., Skirnisdottir, S., Wheat, J.O., Hermannsdottir, A.G. et al. 2005. Isolation and characterization of a thermostable RNA ligase 1 from a Thermus scotoductus bacteriophage TS2126 with good single-stranded DNA ligation properties. Nucleic Acids Res. 33: 135-142.

Cranston, J.W., Silber, R., Malathi, V.G., and Hurwitz, J. 1974. Studies on ribonucleic acid ligase: Characterization of an adenosine triphosphate-inorganic pyrophosphate exchange reaction and demonstration of an enzyme-adenylate complex with T4 bacteriophage-induced enzyme. J. Biol. Chem. 249: 7447-7456.

Deng, J., Schnaufer, A., Salavati, R., Stuart, K.D., and Hol, W.G.J. 2004. High resolution crystal structure of a key editosome enzyme from Trypanosoma brucei: RNA editing ligase 1. J. Mol. Biol. 343: 601-613.

Englert, M. and Beier, H. 2005. Plant tRNA ligases are multifunctional enzymes that have diverged in sequence and substrate specificity from RNA ligases of other phylogenetic origins. Nucleic Acids Res. 33: 388-399.

Fabrega, C., Shen, V., Shuman, S., and Lima, C.D. 2003. Structure of an mRNA capping enzyme bound to the phosphorylated carboxylterminal domain of RNA polymerase II. Mol. Cell 11: 1549-1561.

Filipowicz, W. and Shatkin, A.J. 1983. Origin of splice junction phosphate in tRNAs processed by HeLa cell extract. Cell 32: 547-557.

Gegenheimer, P., Gabius, H.J., Peebles, C.L., and Abelson, J. 1983. An RNA ligase from wheat germ which participates in transfer RNA splicing in vitro J. Biol. Chem. 258: 8365-8373.

Greer, C.L., Peebles, C.L., Gegenheimer, P., and Abelson, J. 1983. Mechanism of action of a yeast RNA ligase in tRNA splicing. Cell 32: 537-546.

Håkansson, K., Doherty, A.J., Shuman, S., and Wigley, D.B. 1997. $\mathrm{X}$-ray crystallography reveals a large conformational change during guanyl transfer by mRNA capping enzymes. Cell 89: $545-553$.

Ho, C.K., Wang, L.K., Lima, C.D., and Shuman, S. 2004. Structure and mechanism of RNA ligase. Structure 12: 327-339.

Laski, F.A., Fire, A.Z., RajBhandary, U.L., and Sharp, P.A. 1983. Characterization of tRNA precursor splicing in mammalian extracts. J. Biol. Chem. 258: 11974-11980.

Martins, A. and Shuman, S. 2004. Characterization of a baculovirus enzyme with RNA ligase, polynucleotide $5^{\prime}$ kinase and polynucleotide $3^{\prime}$ phosphatase activities. J. Biol. Chem. 279: 18220-18231.

Mazumder, R., Iyer, L., Vasudevan, S., and Aravind, L. 2002. Detection of novel members, structure-function analysis and evolutionary classification of the $2 \mathrm{H}$ phosphoesterase family. Nucleic Acids Res. 30: 5229-5243.

Odell, M., Sriskanda, V., Shuman, S., and Nikolov, D. 2000. Crystal structure of eukaryotic DNA ligase-adenylate illuminates the mechanism of nick sensing and strand joining. Mol. Cell 6: 1183-1193.

Pascal, J.M., O’Brien, P.J., Tomkinson, A.E., and Ellenberger, T. 2004. Human DNA ligase I completely encircles and partially unwinds nicked DNA. Nature 432: 473-478.

Phizicky, E.M., Consaul, S.A., Nehrke, K.W., and Abelson, J. 1992. Yeast tRNA ligase mutants are nonviable and accumulate tRNA splicing intermediates. J. Biol. Chem. 267: 4577-4582.
Pick, L. and Hurwitz, J. 1986. Purification of wheat germ RNA ligase: Characterization of a ligase-associated $5^{\prime}$-hydroxyl polynucleotide kinase activity. J. Biol. Chem. 261: 6684-6693.

Pick, L., Furneaux, H., and Hurwitz, J. 1986. Purification of wheat germ RNA ligase: Mechanism of action of wheat germ RNA ligase. J. Biol. Chem. 261: 6694-6704.

Sawaya, R. and Shuman, S. 2003. Mutational analysis of the guanylyltransferase component of mammalian mRNA capping enzyme. Biochemistry 42: 8240-8249.

Sawaya, R., Schwer, B., and Shuman, S. 2003. Genetic and biochemical analysis of the functional domains of yeast tRNA ligase. J. Biol. Chem. 278: 43928-43938.

Schwartz, R.C., Greer, C.L., Gegenheimer, P., and Abelson, J. 1983. Enzymatic mechanism of an RNA ligase from wheat germ. J. Biol. Chem. 258: 8374-8383.

Schwer, B., Sawaya, R., Ho, C.K., and Shuman, S. 2004. Portability and fidelity of RNA-repair systems. Proc. Natl. Acad. Sci. 101: $2788-2793$.

Shuman, S. 2002. What messenger RNA capping tells us about eukaryotic evolution. Nat. Rev. Mol. Cell Biol. 3: 619-625.

Shuman, S. and Lima, C.D. 2004. The polynucleotide ligase and RNA capping enzyme superfamily of covalent nucleotidyltransferases. Curr. Opin. Struct. Biol. 14: 757-764.

Sidrauski, C., Cox, J.S., and Walter, P. 1996. tRNA ligase is required for regulated mRNA splicing in the unfolded protein response. Cell 87: 405-413.

Sriskanda, V. and Shuman, S. 2002a. Role of nucleotidyl transferase motifs I, III and IV in the catalysis of phosphodiester bond formation by Chlorella virus DNA ligase. Nucleic Acids Res. 30: 903-911.

. 2002b. Role of nucleotidyl transferase motif V in strand joining by Chlorella virus DNA ligase. J. Biol. Chem. 277: 9661-9667.

Subramanya, H.S., Doherty, A.J., Ashford, S.R., and Wigley, D.B. 1996. Crystal structure of an ATP-dependent DNA ligase from bacteriophage T7. Cell 85: 607-615.

Sugino, A., Snopek, T.J., and Cozzarelli, N.R. 1977. Bacteriophage T4 RNA ligase: Reaction intermediates and interaction of substrates. J. Biol. Chem. 252: 1732-1738.

Wang, S.P., Deng, L., Ho, C.K., and Shuman, S. 1997. Phylogeny of mRNA capping enzymes. Proc. Natl. Acad. Sci. 94: 9573-9578.

Wang, L.K., Lima, C.D., and Shuman, S. 2002. Structure and mechanism of T4 polynucleotide kinase-An RNA repair enzyme. EMBO J. 21: $3873-3880$

Wang, L.K., Ho, C.K., Pei, Y., and Shuman, S. 2003. Mutational analysis of bacteriophage T4 RNA ligase 1: Different functional groups are required for the nucleotidyl transfer and phosphodiester bond formation steps of the ligation reaction. J. Biol. Chem. 278: $29454-29462$.

Westaway, S.K., Belford, H.G., Apostol, B.L., Abelson, J., and Greer, C.L. 1993. Novel activity of a yeast ligase deletion polypeptide: Evidence for GTP-dependent tRNA splicing. J. Biol. Chem. 268: 2435-2443.

Xu, Q., Teplow, D., Lee, T.D., and Abelson, J. 1990. Domain structure in yeast tRNA ligase. Biochemistry 29: 6132-6138.

Yin, S., Ho, C.K., and Shuman, S. 2003. Structure-function analysis of T4 RNA ligase 2. J. Biol. Chem. 278: 17601-17608.

Zhu, H. and Shuman, S. 2005. Structure-guided mutational analysis of the nucleotidyltransferase domain of Escherichia coli $\mathrm{NAD}^{+}$dependent DNA Ligase (LigA). J. Biol. Chem. (in press).

Zofallova, L., Guo, Y., and Gupta, R. 2000. Junction phosphate is derived from the precursor in the tRNA spliced by the archaeon Haloferax volcanii cell extract. RNA 6: 1019-1030. 

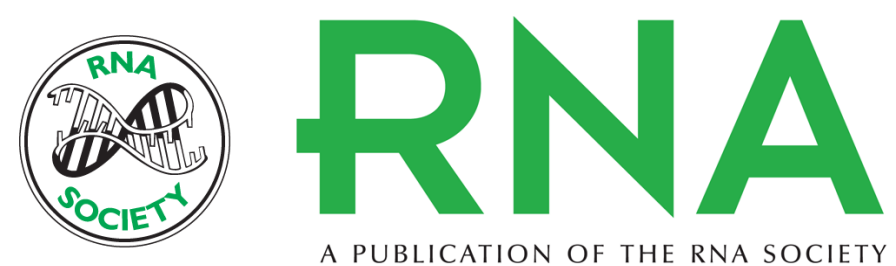

A PUBLICATION OF THE RNA SOCIETY

\section{Structure-function analysis of yeast tRNA ligase}

LI KAI WANG and STEWART SHUMAN

RNA 2005 11: 966-975

References This article cites 39 articles, 20 of which can be accessed free at: http://rnajournal.cshlp.org/content/11/6/966.full.html\#ref-list-1

License

Email Alerting Receive free email alerts when new articles cite this article - sign up in the box at the Service top right corner of the article or click here. 